\title{
Acrophobia treatment with virtual reality-assisted acceptance and commitment therapy: two case reports
}

\author{
Zulal Celik $^{\circledR}$, Furkan Bahadir Alptekin ${ }^{2}{ }^{\circledR}$, Kaasim Fatih Yavuz ${ }^{3}$ \\ ${ }^{1}$ Karaman State Hospital, Department of Psychiatry, Karaman - Turkey \\ ${ }^{2}$ Kurtalan State Hospital, Department of Psychiatry, Siirt - Turkey \\ ${ }^{3}$ Istanbul Medipol University, Department of Psychology, Istanbul - Turkey
}

\begin{abstract}
Exposure has an essential place in the treatment of acrophobia and technology may increase the success of exposure therapies in many ways. We predicted that the integration of acceptance and commitment therapy (ACT) with Virtual Reality (VR) technology would be beneficial in the treatment of acrophobia and would improve clients' compliance with therapy. This report presents two examples of VR-assisted ACT treatment for acrophobia. The process consists of six sessions (one initial interview and five sessions of interventions) for individual therapy. In the first two sessions, functional behavioral analysis and case formulation with clients were performed and skills to be used during exposure sessions taught. From the second session, application of VR-assisted exposure interventions with ACT principles was started. Results showed a significant improvement for both clients. At the end of the treatment, they were both able to complete the virtual behavioral approach task and presented decreased avoidance strategies and increased value-based behaviors. VR-assisted ACT is promising, especially to facilitate exposure treatments.
\end{abstract}

Keywords: Acceptance and commitment therapy, acrophobia, exposure, virtual reality

\section{INTRODUCTION}

Acrophobia, which is an extreme fear of heights, is a specific natural environment type phobia. It is the most common variety of specific phobias affecting males, and the second or third most common type in females (1). While physiological visual height imbalance in fearsome environments and situations such as towers, staircases, bridges, cliffs, or mountain ridges may affect $100 \%$ of people (2), individuals with acrophobia react differently. Although the incidence in the community is high, only a small percentage of affected persons seek professional help (3).
Most studies and reviews published in recent years emphasize that cognitive and behavioral therapies are the most effective methods in the treatment of specific phobias, including acrophobia (2). A recent metaanalysis of 16 studies compared five broad categories of intervention: desensitization (DS); in vivo exposure (IVE); virtual reality exposure (VRE); neuro-linguistic programming, and VRE with medication. There is empirical research demonstrating the effectiveness of IVE, DS, and VRE in the short term (4).

VRE is used in a wide range of psychotherapy treatments including for PTSD, anxiety disorders (5), psychotic disorders (6), substance use disorders, and

How to cite this article: Celik Z, Alptekin FB, Yavuz KF. Acrophobia treatment with virtual reality-assisted acceptance and commitment therapy: two case reports. Dusunen Adam The Journal of Psychiatry and Neurological Sciences 2020;33:317-324.

Correspondence: Furkan Bahadir Alptekin, Kurtalan State Hospital, Department of Psychiatry, Siirt - Turkey 
eating disorders (7). For the first time in 1995, Rothbaum et al. (8) demonstrated the efficacy of VRE therapy (VRET) in acrophobia. VRET can successfully reveal fear and anxiety and it saves time and money by allowing us to conduct a session in the therapy room. It also provides a safer environment for clients by removing risks such as insecurity and shame that may arise in public spaces. With these advantages, rejection of treatment by clients is decreasing (9).

Acceptance and commitment therapy (ACT) is a form of cognitive-behavioral therapies that have acquired growing evidence for the treatment of broad clinical problems. A review published in 2014 showed that ACT has moderate evidence for mixed anxiety disorders, generalized anxiety disorder, obsessivecompulsive disorder (OCD), and social phobia (10). While there is no controlled study in the field of specific phobia interventions with ACT, to date there is only one laboratory-based study of ACT used in arachnophobia. It was found that the ACT group was able to go further and more prepared to perform the same procedure the week following therapy (11). In this report, the VR-assisted ACT process of two cases with acrophobia will be presented in detail and the findings will be discussed in the light of the literature.

\section{CASES}

\section{Participants and Therapists}

Two clients suffering from acrophobia participated in the therapy. They both met the DSM- 5 criteria for the diagnosis of acrophobia. The clients' level of acrophobia was assessed and confirmed by clinical interview. The clients' names were changed to protect their privacy and both signed an informed consent form.

The therapists (first two authors) are practicing as psychiatrists (MD) in a mental hospital. All therapists are trained in ACT and supervised by an ACT trainer and supervisor (third author).

\section{Technical Equipment and Software}

The VR equipment consisted of an Oculus Rift Virtual Reality headset and Oculus Touch Controllers (http:// www.oculus.com). "Safemind" is a virtual reality-based anxiety and phobia treatment platform for mental health professionals that enables them to implement exposure therapy in their treatment sessions (www.safemind.app).

\section{Measures}

The Acceptance and Action Questionnaire-II (AAQ-II) assesses the differences in experiential avoidance and psychological inflexibility among individuals. It is a 7-point Likert-type self-report scale (1: never true, 7: always true) with higher scores indicating higher levels of experiential avoidance and psychological rigidity (12). A Turkish validity and reliability study of the AAQ-II was performed by Yavuz et al. (13).

The Simulator Sickness Questionnaire (SSQ) is a 16-item self-report questionnaire (14). The SSQ measures cybersickness symptoms like nausea, oculomotor disturbances, and disorientation in the context of simulation (15). It is the most commonly used scale for simulator sickness.

The Acrophobia Assessment Questionnaire (AcAQ) was developed based on the Fear Questionnaire (FQ) (16) to evaluate height-related avoidance, disturbing and accompanying symptoms. The AcAQ has been specifically developed by the authors with regard to acrophobia and consists of three 8-point Likert-type subscales (for avoidance, disturbing, and accompanying symptoms, respectively).

The Igroup Presence Questionnaire (IPQ) is a 14-item 5-point Likert-style self-report measure. The IPQ was developed to measure the sense of presence experienced in the context of simulation (17) and consists of three subscales (Spatial Presence: the sense of being physically present, Involvement: measuring the involvement level, Experienced Realism: measuring the subjective experience of realism) and an additional item for measuring global presence not loaded onto subscales $(17,18)$

The Subjective Units of Distress Scale (SUDS) assesses the level of disturbance or distress currently experienced. SUDS is a self-report scale and the individuals report their current anxiety in a range between 0 (no distress) and 100 (highest distress) (19). In this study, the SUDS was used as a measure of experienced anxiety in each session.

The Subjective Units of Willingness Scale (SUWS), a visual analog scale prepared by the authors for the assessment of state willingness, is a self-report instrument assessing the level of willingness to experience difficult thoughts and feelings. Subjects report their current openness/acceptance on a scale between 0 and 100. Willingness levels are assessed in every session throughout the therapy.

The Acrophobia Questionnaire (AQ) is a 40-item self-report scale assessing individuals' anxiety and avoidance levels associated with 20 height-relevant situations (20). For the needs of the current study, only 20 items of the AQ related to the anxiety level were used. 
The Behavioral Approach Test (BAT) is an objective measure to evaluate how close the participant is to the feared object or situation. With BAT, it is possible to measure both the severity of the phobia and the effectiveness of the therapy (21). However, there is no standard BAT application (22). While the BAT is generally used in a real environment, there are also studies involving its use in a virtual environment $(2,23)$. In this study, we used the Virtual Reality Behavior Avoidance Test (VR-BAT). In our cases, we asked our clients to go up by outdoor elevator to the highest floor of a skyscraper in a virtual environment, and we calculated the tolerated maximum time and height of ascent. Clients took the VR-BAT in the pre-treatment session (initial interview) and at the end of the 5th session.

\section{Program Overview}

In total, six sessions including an initial interview were planned on a weekly basis. The duration of each session was 45 minutes. Initial interviews were conducted for assessment. The clients were informed about virtual reality-assisted ACT for acrophobia and their signed informed consent was obtained. Detailed content of every session is shown in Table 1 .

\section{Case 1}

A 29-year-old female medical doctor presented to our clinic for height phobia. Her fear of heights had been present from a young age. She could not go on highaltitude thrill rides when she went to the amusement park in primary school. In high places - especially when they were not secured with railings - there was a feeling of fear, anxiety, and unease; at that time, she thought that she could fall to her death, and then she would step back and sometimes scream. In the absence of a parapet, she could not look down from a 2 nd-floor balcony. If there was a barrier, on a 3rd-floor balcony, she could stand one step back from the edge; on the 5th and 6th floors, she could only keep her back against the rear wall. She could visit tourist attractions with her friends, but she could not go to high places. Although this problem did not cause negative consequences in interpersonal relations, it reduced her quality of life. The client met the "height phobia" criteria of DSM-5 according to clinical interview, and no comorbid psychiatric disorder was found. She could not complete BAT and stopped the elevator after 20 seconds (at approximately 35 feet).

In the first session, the ACT matrix (24) was used to formulate the client's problem. Behaving like a brave person, participating in exciting activities, and freedom were identified as values related with height phobia. The client wanted to do bungee jumping, skydiving, and participate in similar activities, but said she avoided these situations because of experiencing many unwanted emotions, sensations, and thoughts every time she tried to engage in these activities. After working on establishing willingness, it was then discussed how to deal with unwanted private experiences in high places.

Subsequently, the client entered a scene in the virtual environment, a terrace of a high building. While she approached the edge of the terrace, tension, trembling in the hands, and a desire to escape emerged. She was taught grounding and acceptance of emotions instead of escaping when feeling anxiety. Daily grounding and acceptance exercises were planned between sessions. VR-assisted exposure was started in session 2. In this session, it was realized that she was waiting for the uncomfortable feelings to end in order to get to higher places. At the beginning of the 3rd session, creative hopelessness intervened once again. Afterwards, it seemed that willingness to experience uncomfortable feelings on the elevator had increased. VRET was applied in sessions 3, 4, and 5. At the end of the 5th session, she could reach the highest point in the virtual environment by elevator, so she completed the BAT. Within the period of therapy she also moved to a higher-rise building and listened to a concert on the third floor with her friend. She reported that she also had the desired fun when she attended the concert.

\section{Case 2}

The patient was a 45-year-old Turkish female gynecologist with her own private practice living in Istanbul with her husband and three children. She recalled that her acrophobia began when she was driving a car thirteen years ago. The spaces she feared most were high bridges on main roads, high-rise buildings, steep hills, and wide-open fields. She especially could not look down once she reached the 6th floor of a building. In those situations, she recalled feeling very intense fear and anxiety. For 13 years, her complaints had been increasing and decreasing from time to time. Currently, she still encounters difficulties when ascending a building, crossing a high bridge on her own, driving a car by herself, or boarding a plane without taking medication. Even if it requires a long detour, she tries to find routes without bridges or high places.

The client met the full criteria for DSM-5 "height phobia" according to a clinical interview. She stayed for 
Table 1: Content of sessions

ACT key concepts

Initial interview $\quad-$ Introduction of VR-assisted ACT therapy
- Identifying ACT-consistent
treatment alliances and goals

$1^{\text {st }}$ session

$2^{\text {nd }}$ to $5^{\text {th }}$ session

\author{
-Using the ACT Matrix (24) for: \\ - Identifying values \\ - Identifying height-related private events \\ (thoughts, emotions, bodily sensations) \\ - "Control is the problem" \\ - Creative hopelessness \\ - Choosing a valued direction \\ - Valued action \\ - Learning new skills \\ - Acceptance \\ Grounding
}

\begin{abstract}
- Virtual Reality-assisted Exposure
( $2^{\text {nd }}$ session: 20 minutes, $3^{\text {rd }}, 4^{\text {th }}$,
\end{abstract}

and $5^{\text {th }}$ sessions: $30-35$ minutes)

- Learning how to move with private events

- Practicing new skills (applying acceptance, willingness, grounding and defusion

to stay with discomfort)

- Valued action

\section{Purposes}

- Informing the client about the therapy process.

- Taking the client's history and establishing

a therapeutic relationship with the client.

- Identifying ACT-consistent treatment goals.

- Introduction of virtual reality equipment and how to use the headset.

- Testing VR in a neutral scenario.

- Implementation of the VR-BAT

- Client identifies values and determines value-based life directions.

- Client learns to observe his or her private events

- Client identifies his/her coping styles for these difficult private events

- Client learns that the coping strategies he/she used are for controlling difficult private events. Therapist validates client's private events and struggle with them.

- Client learns in a validated way that these strategies are impeding his/her life functioning.

- Client experiences symptoms in virtual environment (virtual exposure)

- Client learns acceptance and grounding, how to stay with discomfort, as an alternative to avoidance.

- Client chooses to be open and willing to confront his/her difficult private events. He/she learns to move in a valued direction with them instead of avoiding them.

- Client experiences symptoms without avoidance behaviors (virtual exposure)

- Client learns acceptance, how to stay with discomfort, as an alternative to avoidance.

- Client learns to observe private events and chooses to be open and willing to experience them even if they are difficult for him/her.

- Client learns to be directed by his/her values rather than by difficult private events.
21 seconds (approximately 37 feet) in the VR-BAT test conducted in the initial interview. In the first session, the ACT matrix (24) was performed to assess the state of the client through a functional approach. She had thoughts like "I'll fall," "it is not safe" and several self-critical thoughts like "why are you afraid?", "you always do that, why can't you do it?" She reported feeling a sense of inadequacy, intense fear, and anxiety including bodily sensations such as severe tachycardia, sweating, numbness below the knees, and difficulty in breathing. The client identified the techniques she used to cope with these uncomfortable internal experiences (postpone plans, making long detours, driving with someone next to her in the car). Creative hopelessness enabled the client to realize that the coping strategies she had used made her more desperate and tired. She also admitted that this 
state distanced her from her values to lead a meaningful life (freedom, self-improvement, seeing new places, helping people) that remained important to her. Acceptance and grounding exercises allowed the client to realize that she could also get through painful private events, and if she opened herself to experiencing these uncomfortable situations, she could live according to her own needs and values rather than spending time trying to avoid them. She found defusion exercises particularly useful because they allowed her to notice self-critical thoughts that always responded to each other in her mind. In addition to increasing willingness and acceptance to experience her height-related bodily sensations and emotions, she said that being aware of critical thoughts, though not a direct target of therapy, helped her in other areas of her life. VRET was applied in sessions 3, 4, and 5. Although not assigned as homework, after the 3rd session she drove a car alone and crossed the Bosphorus Bridge several times. At the end of the therapy, she completed VR-BAT and reached the highest point in the virtual environment by elevator. Even if her heightrelated anxiety symptoms were changing from time to time, instead of delaying action until fear and anxiety were eliminated, she began to move towards her goals that were related to ensuring a meaningful life. She began to drive a car alone, organize trips with her family without obsessing over the height of the place they would choose, and cross high bridges alone several times with her anxiety symptoms being present.

Both clients had low SSQ scores, which shows that the severity of cybersickness was very low. The three subscales of IPQ assess the components of presence (17). Total IPQ scores decreased from 62 to 53 for case 1 and increased from 31 to 61 for case 2 at the end of the treatment. Higher IPQ scores indicate a greater sense of presence (25).

SUDS (0-100) and willingness scores (0-10) were measured before, every 3-5 minutes during, and at the end of the exposure. At the end of the treatment, both clients' SUDS points were reduced and willingness points increased. Both clients' VR-BAT scores equally increased at the end of the treatment. Both of the clients completed the VR-BAT.

The AcAQ was evaluated separately for each subscale. At six months after the end of the treatment, the avoidance score declined from 4 to 2 for case 1 and from 7 to 4 for case 2 (Figure 1). The level of disturbing score also decreased from 7 to 2 for case 1 and from 6 to 5 for case 2 (Figure 2). Finally, the level of accompanying symptoms went down from 27 to 6 for case 1 and from 35 to 18 for case 2 (Figure 3 ).

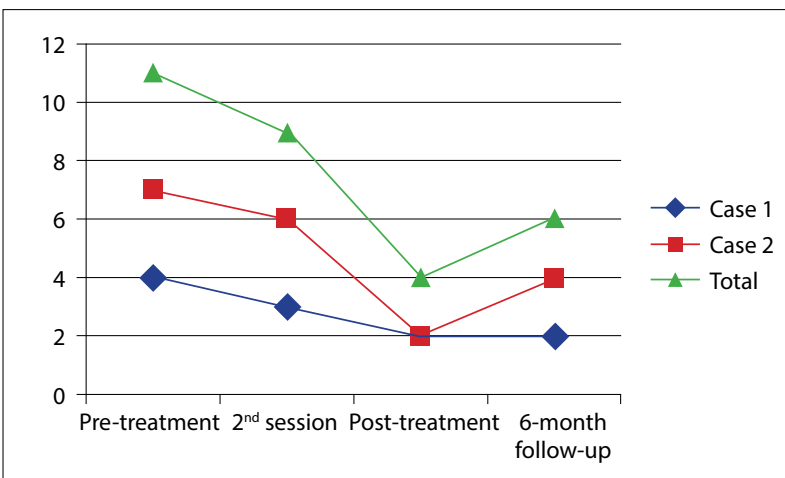

Figure 1. Changes in the avoidance subscale levels of the Acrophobia Assessment Questionnaire.

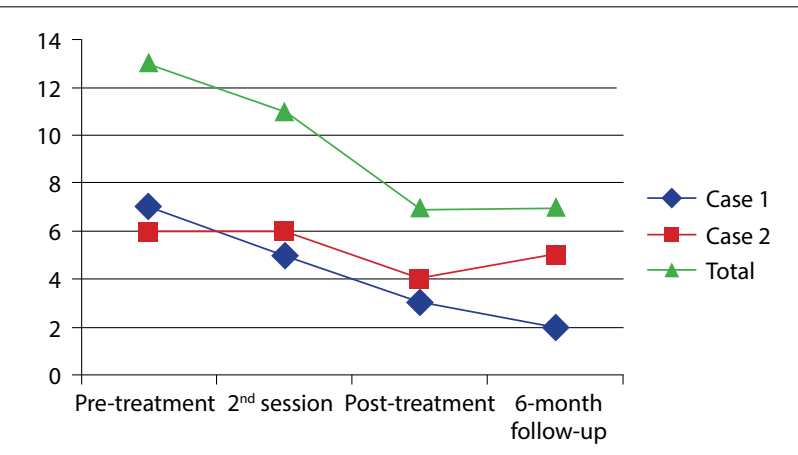

Figure 2. Changes in the disturbing subscale levels of the Acrophobia Assessment Questionnaire.

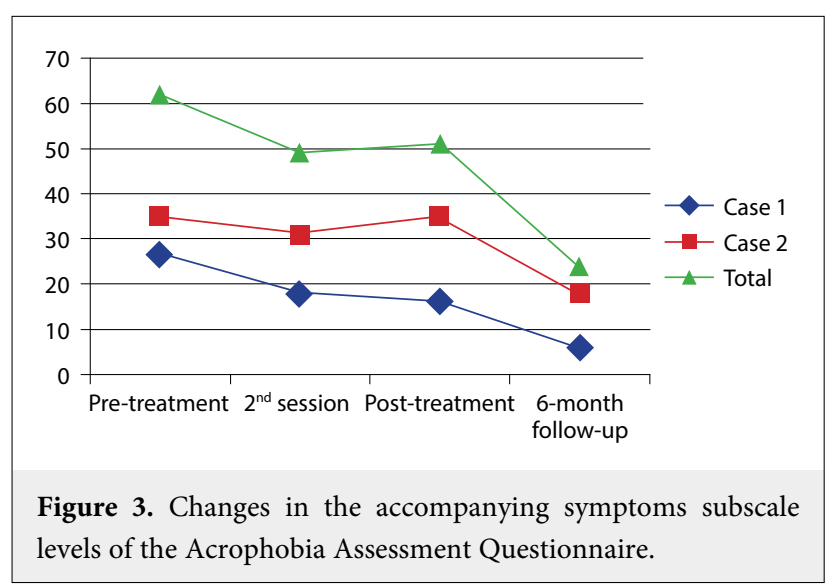

The course of anxiety subscores of AQ was different for each client. For case 1, the AQ score was reduced from 46 to 12 after treatment and was 22 at six-month follow-up. For case 2, the AQ score increased from 26 to 36 post-treatment and the six-month follow-up AQ score was 37 (Figure 4).

The trajectories of the two clients' AAQ-II scores were different from each other. For case 1, the AAQ-II score decreased from 21 to 8 , and for case 2 , it increased from 28 to 32 post-treatment and decreased to 28 again at six-month follow-up (Figure 5). 

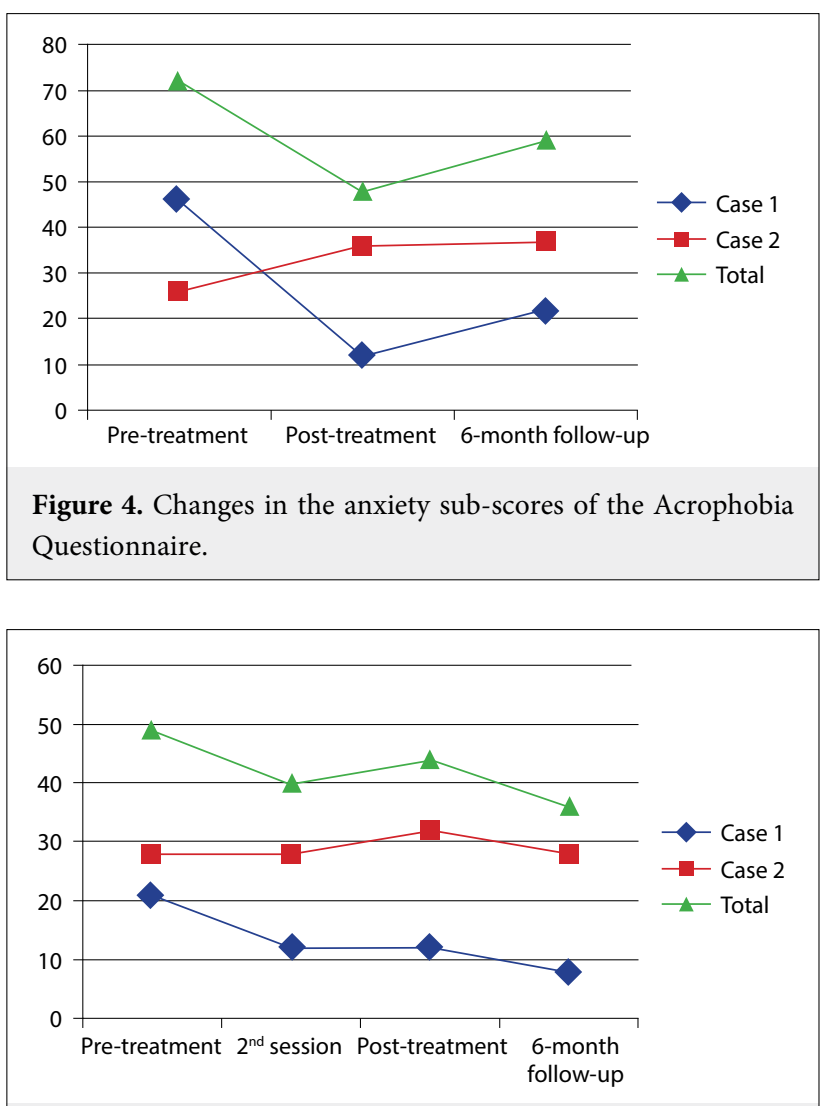

Figure 5. Changes in the Acceptance and Action Questionnaire-II.

\section{DISCUSSION}

Virtual reality is increasingly used in theory development, assessment, and treatment of various clinical conditions. The main areas of use are anxiety disorders, psychosis/ schizophrenia, substance use disorders, eating disorders, and autistic spectrum disorders (26). There is also research on the treatment of social cognition, social skills training, motor learning, verbal communication, phobias, and attention deficit in the assessment of the autistic spectrum (27). This report aimed to show preliminary data of the effectiveness of a Virtual Reality-Assisted ACT program for acrophobia. In addition to effectiveness studies (28), there are many randomized controlled trials of ACT that include exposure techniques implemented in several diagnostic groups such as OCD (29), social anxiety (30), and mixed anxiety groups, and findings suggested that ACT is an effective approach for exposurebased interventions. However, the number of studies with ACT for specific phobias is limited (11), and there is no previous research on the effectiveness of ACT for acrophobia as far as we can determine.

VR technology - used in the treatment of exposure in the last three decades - has increased its use and prevalence both in treatment and research of psychopathology. However, VR-assisted ACT studies have just begun. At present, there is only one study conducted by Prudenzi et al. (31) who applied a VR task for cognitive defusion to investigate the impact of VR on individuals' relations with negative self-referential thought. They found that the VR-assisted defusion intervention reduced the believability and negativity and increased comfort and willingness regarding disturbing thoughts. This is the first report that involves ACT and VRET together.

From the ACT perspective, the purpose of exposure is to focus on acceptance of inner experiences rather than reduce or control them and to help clients act in a more functional and flexible way in accordance with their values (32). Contrary to traditional, habituation-based exposure methods, the ACT approach is not focused on fear reduction (32). ACT emphasizes helping clients live a valuable life by accepting disturbing private events such as fear and anxiety rather than avoiding them. In the context of values, the function of fear and anxiety-related stimuli are transformed and the client becomes more open to aversive experiences (33). Following sessions in the light of these principles, although clients were able to reach respectively 35 and 37 feet before exposure, both of the clients could complete the VR-BAT at the end of the therapy (which means they reached the highest point, approximately 212 feet). Additionally, these results were consistent with the clients' everyday life.

At the end of the treatment, the Acrophobia Assessment Questionnaire (AcAQ) level of avoidance and level of disturbance subscale scores decreased for both clients. At six-month follow-up, both clients' avoidance and disturbance levels remained the same or continued to decrease. While the first client's psychological inflexibility score continued to decrease, the second client's psychological inflexibility score increased and then remained at the same level as the pretreatment score. Likewise, the first client's anxiety level was lower compared to pre-treatment, whereas the second client's anxiety level increased compared to pretreatment assessment. Even though the anxiety level of the second case increased, her avoidant behaviors were reduced and value-oriented actions increased. From the ACT perspective, it can be said that even in the presence of feared stimuli, new actions (sharing with family, selfimprovement) could be elicited when they were connected with their values. Through this process, clients willingly learn to acknowledge their anxiety symptoms and do not try to suppress them. As a result, in the ACT process it is not rare to see that anxiety symptoms still exist; however, they lose their effect on clients' behaviors. 
While the first client's AAQ-II total score decreased during the therapy, the second client's score increased. Inconsistency between AAQ-II scores and behavioral measures in the second case may be due to the subjective feature of the AAQ-II. Another explanation could be that the AAQ-II was designed for measuring general psychological inflexibility levels, not specific to acrophobia. Development of phobia-specific psychological inflexibility measures may be more suitable for future studies.

Performing the BAT in a virtual environment may be a limitation of this study. However, there are some studies for which successful data exist with BAT being performed in a virtual environment $(2,23)$. In addition, both our clients' experiences between sessions were consistent with the VR-BAT assessments. Further studies need to be done for comparisons of real-life and VR-BAT assessment. Another limitation is the absence of Turkish validity and reliability studies for the SSQ, AcAQ, IPQ, SUDS, SUWS, AQ, and BAT.

To conclude, in our cases ACT for acrophobia treatment with VR has been effective in reducing their height-related avoidance and helping them take steps in line with their values. In the context of acrophobia, both clients' value-based actions continued to increase at sixmonth follow-up. Taken together, these results indicate that effects of the therapy were still persisting after six months. However, it is necessary to expand the VR-assisted ACT studies with RCTs to test these preliminary findings. New study designs are also needed in the treatment of height phobia to compare VR-assisted ACT to other therapies using VR. Performing additional studies that investigate the relation between VR-BAT and in vivo BAT would also be beneficial. Future studies should investigate evidence for the effectiveness of VR-assisted ACT in specific phobias, particularly in acrophobia.

\begin{tabular}{|c|c|c|}
\hline \multicolumn{2}{|c|}{ Contribution Categories } & Author Initials \\
\hline \multirow{4}{*}{ Category 1} & Concept/Design & K.F.Y. \\
\hline & Literature review & Z.C., F.B.A. \\
\hline & Data analysis/Interpretation & Z.C., F.B.A. \\
\hline & Case follow-up (if applicable) & Z.C., F.B.A. \\
\hline \multirow{2}{*}{ Category 2} & Drafting manuscript & Z.C., F.B.A. \\
\hline & Critical revision of manuscript & K.F.Y. \\
\hline Category 3 & Final approval and accountability & K.F.Y., Z.C., F.B.A. \\
\hline \multirow{2}{*}{ Other } & Technical or material support & K.F.Y. \\
\hline & Supervision & K.F.Y. \\
\hline
\end{tabular}

Informed Consent: Written consent was obtained from the patients. Peer-review: Externally peer-reviewed.
Conflict of Interest: The authors have not stated any conflict of interest.

Financial Disclosure: The authors have not received any financial support for this study.

\section{REFERENCES}

1. LeBeau RT, Glenn D, Liao B, Wittchen HU, Beesdo-Baum K, Ollendick T, et al. Specific phobia: a review of DSM-IV specific phobia and preliminary recommendations for DSM-V. Depress Anxiety 2010; 27:148-167.

2. Brandt T, Huppert D. Fear of heights and visual height intolerance. Curr Opin Neurol 2014; 27:111-117.

3. Magee WJ, Eaton WW, Wittchen HU, McGonagle KA, Kessler RC. Agoraphobia, simple phobia, and social phobia in the National Comorbidity Survey. Arch Gen Psychiatry 1996; 53:159168.

4. Kapfhammer HP, Fitz W, Huppert D, Grill E, Brandt T. Visual height intolerance and acrophobia: distressing partners for life. J Neurol 2016; 263:1946-1953.

5. Opris D, Pintea S, García-Palacios A, Botella C, Szamoskozi S, David D. Virtual reality exposure therapy in anxiety disorders: a quantitative meta-analysis. Depress Anxiety 2012; 29:85-93.

6. Freeman D, Garety P. Advances in understanding and treating persecutory delusions: a review. Soc Psychiatry Psychiatr Epidemiol 2014; 49:1179-1189.

7. Hone-Blanchet A, Wensing T, Fecteau S. The use of virtual reality in craving assessment and cue-exposure therapy in substance use disorders. Front Hum Neurosci 2014; 8:844.

8. Rothbaum BO, Hodges LF, Ready D, Graap K, Alarcon RD. Virtual reality exposure therapy for Vietnam veterans with posttraumatic stress disorder. J Clin Psychiatry 2001; 62:617-622.

9. Garcia-Palacios A, Botella C, Hoffman H, Fabregat S. Comparing acceptance and refusal rates of virtual reality exposure vs. in vivo exposure by patients with specific phobias. Cyberpsychol Behav 2007; 10:722-724.

10. Bluett EJ, Homan KJ, Morrison KL, Levin ME, Twohig MP. Acceptance and commitment therapy for anxiety and OCD spectrum disorders: an empirical review. J Anxiety Disord 2014; 28:612-624.

11. Wagener AL, Zettle RD. Targeting fear of spiders with control-, acceptance-, and information-based approaches. Psychol Rec 2011; 61:77-91.

12. Bond FW, Hayes SC, Baer RA, Carpenter KM, Guenole N, Orcutt HK, et al. Preliminary psychometric properties of the Acceptance and Action Questionnaire-II: a revised measure of psychological inflexibility and experiential avoidance. Behav Ther 2011; 42:676-688.

13. Yavuz F, Ulusoy S, Iskin M, Esen FB, Burhan HS, Karadere ME, et al. Turkish version of acceptance and action questionnaire-II (AAQ-II): a reliability and validity analysis in clinical and non clinical samples. Klinik Psikofarmakol Bulteni 2016; 26:397-408.

14. Kennedy RS, Lane NE, Berbaum KS, Lilienthal MG. Simulator sickness questionnaire: an enhanced method for quantifying simulator sickness. Int J Aviat Psychol 1993; 3:203-220. 
15. Kellogg RS, Kennedy RS, Graybiel A. Motion sickness symptomatology of labyrintine defective and normal subjects during zero gravity maneuvers. Aerosp Med 1965; 36:315-318.

16. Marks IM, Mathews AM. Brief standard self-rating for phobic patients. Behav Res Ther 1979; 17:263-267.

17. Schubert T, Friedmann F, Regenbrecht H. The experience of presence: factor analytic insights. Presence 2001; 10:266-281.

18. Schubert TW. The sense of presence in virtual environments: a three-component scale measuring spatial presence, involvement, and realness. J Media Psychol 2003; 15:69-71.

19. Wolpe J. The Practice of Behavior Therapy. Fourth ed., New York: Pergamon Press, 1990.

20. Cohen DC. Comparison of self-report and overt-behavioral procedures for assessing acrophobia. Behav Theraphy 1977; 8:1723.

21. Choy Y, Fyer AJ, Lipsitz JD. Treatment of specific phobia in adults. Clin Psychol Rev 2007; 27:266-286.

22. Arroll B, Wallace HB, Mount V, Humm SP, Kingsford DW. A systematic review and meta-analysis of treatments for acrophobia. Med J Aust 2017; 206:263-267.

23. Tart CD, Handelsman PR, Deboer LB, Rosenfield D, Pollack MH, Hofmann SG, et al. Augmentation of exposure therapy with postsession administration of D-cycloserine. J Psychiatr Res 2013; 47:168-174.

24. Polk KL, Schoendorff B, Webster M, Olaz FO. The Essential Guide to the ACT Matrix: A Step-by-Step Approach to Using ACT Matrix Model in Clinical Practice. Oakland, CA: New Harbinger Publications, 2016, 19-40.

25. Price M, Mehta N, Tone EB, Anderson PL. Does engagement with exposure yield better outcomes? Components of presence as a predictor of treatment response for virtual reality exposure therapy for social phobia. J Anxiety Disord 2011; 25:763-770.
26. Kim S, Kim E. The Use of Virtual Reality in Psychiatry: A Review. Soa Chongsonyon Chongsin Uihak 2020; 31:26-32.

27. Miller IT, Wiederhold BK, Miller CS, Wiederhold MD. Assessment and treatment of autism spectrum disorders with virtual reality: a comprehensive research chart. Cyberpsychol Behav Soc Netw 2020; 23:60-65.

28. Etchemendy E, Banos RM, Botella C. Virtual and augmented reality as useful and efficacious tools for the psychological treatment of emotional disorders: In Cipresso $\mathrm{P}$, Serino $\mathrm{S}$ (editors). Virtual Reality: Technologies, Medical Applications and Challenges. New York: Nova Publishers, 2015, 31-54.

29. Arch JJ, Eifert GH, Davies C, Plumb Vilardaga JC, Rose RD, Craske MG. Randomized clinical trial of cognitive behavioral therapy (CBT) versus acceptance and commitment therapy (ACT) for mixed anxiety disorders. J Consult Clin Psychol 2012; 80:750-765.

30. Craske MG, Niles AN, Burklund LJ, Wolitzky-Taylor KB, Vilardaga JC, Arch JJ, et al. Randomized controlled trial of cognitive behavioral therapy and acceptance and commitment therapy for social phobia: outcomes and moderators. J Consult Clin Psychol 2014; 82:1034-1048.

31. Prudenzi A, Rooney B, Presti G, Lombardo M, Lombardo D, Messina $\mathrm{C}$, et al. Testing the effectiveness of virtual reality as a defusion technique for coping with unwanted thoughts. Virtual Reality 2019; 23:179-185.

32. Meuret AE, Twohig MP, Rosenfield D, Hayes SC, Craske MG. Brief acceptance and commitment therapy and exposure for panic disorder: a pilot study. Cogn Behav Pract 2012; 19:606618.

33. Morris E. So long to SUDs - Exposure is not about fear reduction... it's about new learning and flexibility http:// drericmorris.com/2017/01/13/nosuds/. Accessed December 6, 2019. 